\section{Co-directores}

Marc Antoni Broggi i Trias (PCBC)

Francesc Borrell (UB)

\section{Jefa de Redacción}

Núria Estrach (UAB)

\section{Consejo científico}

Juan Carlos Hernández Clemente

Juan Medrano Albéniz

Vicente Morales Hidalgo

\section{Correspondencia}

Web:

http://www.fundacionletamendi.com

Correo electrónico:

info@fundacionletamendi.com

\section{Envío de manuscritos:}

http://www.fundacionletamendi.com/revista-foliahumanistica/envio-de-manuscritos/

\begin{abstract}
Información editorial
Folia Humanística publica artículos por encargo solicitados a especialistas, así como aquellas propuestas enviadas por los autores y aceptadas tras su evaluación por pares de académicos especializados.

Los textos recibidos se publicarán en la lengua original (castellano, catalán, inglés y francés); los que se consideren de relevancia mayor serán traducidos al inglés y castellano.

Los artículos deben ser originales y acompañados del documento "derechos de autor" que encontrarán en la web, junto a las normas de presentación a seguir.

Cada artículo publicado al final tendrá especificado la referencia de citación, donde se incluirá el número DOI ${ }^{\circledR}$.

\section{Distribución \\ La Revista Folia Humanística es de libre acceso a consultar online. \\ http://www.fundacionletamendi.com/category/rev istal}

Folia Humanística es una revista internacional que tiene el doble objetivo de fomentar, por un lado, la reflexión y el debate público en el ámbito de la Salud, Ciencias Sociales y Humanidades, y por el otro, la colaboración entre distintos equipos de investigación nacionales e internacionales que dinamicen el diálogo entre la filosofía de la medicina, la salud pública y la justicia social. Dividida en "Tema del día", (artículos para el debate), "Pensamiento actual", (artículos críticos de novedades editoriales), y "Arte, Salud y Sociedad", la revista se esfuerza en fortalecer las conexiones entre la investigación académica, la práctica clínica, las experiencias de los pacientes y sus implicaciones éticas y estéticas en la sociedad. Todo ello con la intención de favorecer la reflexión entre diferentes disciplinas sobre temas de actualidad y las tendencias más novedosas en el campo de las Humanidades y la Salud.

Folia Humanística is an International Journal, born with the dual aim of fuelling the discussion and public debate on issues of health, social sciences and humanities and on the hand, of fostering cooperation between various research groups, both national and International, to spur the dialogue between philosophy and medicine, public health and social justice. The Journal is divided into three different sections: "main focus" (article for debate), "Contemporary thought" (critical reviews of new Publications) and "Arts, Health and Society" which all contribute to strengthening the links between academic research, clinical practice, the experience of patients and their ethical and esthetical implications for society. Ultimately, the intention of the Journal is to promote reflection at the crossroads of several disciplines on topical issues and new trends in humanities and health. 


\title{
RE-APRENDER A PENSAR. LA URGENCIA DE FORMARSE EN EL PENSAMIENTO CRÍTICO COMO PROFESIONALES Y COMO CIUDADANOS.
}

\section{JOAN RUÉ DOMINGO}

\begin{abstract}
Resumen: La complejidad de muchos fenómenos que, a diario, se nos presentan, nos interpelan como ciudadanos, sin que tengamos las claves para su comprensión y eventual resolución, a pesar de unos sistemas educativos con altas cotas de escolarización y de acreditación en los estudios. Ello interpela a los modelos de formación y de aprendizaje vigentes dentro del sistema educativo, en la medida que las personas se acreditan sin los recursos para pensar críticamente y reflexionar sobre sí mismos, sobre las formas de vida y la misma democracia. El texto destaca la necesidad urgente de enseñar a pensar de modo crítico en todos los escenarios de la formación sin distinguir las necesidades formativas de la profesionalidad de las personales. Aprender a pensar de modo crítico es educarse. Pero ello no será posible si antes no analizamos nuestras propias limitaciones y no atendemos las claves básicas necesarias para hacerlo posible
\end{abstract}

Palabras clave: pensamiento crítico/ formación/ ciudadanía/ democracia/ empoderamiento.

\begin{abstract}
:
The complexity of many of daily phenomena defies our comprehension as citizens, due to the lack of the keys to their comprehension and eventual resolution, despite our educational systems with high levels of schooling and accreditation in studies. This challenge the models of training and learning within the education system, as people are credited without the basic resources to think critically and to reflect on themselves, on the way they live and on democracy itself. The text highlights the urgent need to teach how to think critically in all scenarios of training without distinguishing the formative needs of professionalism from the personal ones. Learning to think critically is about education itself. But this will not be possible if we do not analyze first our own limitations and do not attend to the basic keys that make it possible.
\end{abstract}

Keywords: critical thinking/training/ citizenship/ democracy/ empowerment.

Artículo recibido: 7 noviembre 2018; aceptado: 8 marzo 2019.

\section{INTRODUCCIÓN}

Existe un amplio consenso en el hecho de que, en los tiempos actuales, tres grandes fenómenos convergen en una escala que es nueva en la historia y que nos afectan a los ciudadanos y a las sociedades de manera significativa. En primer lugar, la alta complejidad de los muchos fenómenos que, a diario, se nos presentan y nos interpelan como ciudadanos, sin que tengamos sus verdaderas claves tanto para su comprensión como para su abordaje y eventual resolución. En segundo lugar, el 
Re-aprender a pensar. La urgencia de formarse en

el pensamiento crítico como profesionales y como ciudadanos.

Joan Rué Domingo

sentimiento de impotencia de las personas, al entender que las potenciales soluciones no sólo se encuentran fuera de su alcance, sino que les afectarán negativamente. Finalmente, en contradicción con lo anterior: determinados modelos de aprendizaje aún muy vigentes dentro del sistema educativo, y también muchos poderes que quieren ejercer su influencia en la sociedad, proponen el mensaje de que cualquier hecho tiene solución; o bien que muy a menudo estas soluciones obedecen a una lógica simple y que todo puede ser abordado y comunicado en términos de entretenimiento.

Esta ecuación propicia en los ciudadanos la rendición del pensamiento y de su comportamiento democrático a cambio de asumir prejuicios y aceptar unas pretendidas seguridades que les son ofrecidas, poniendo en riesgo, en última instancia, el propio concepto de democracia. Marina Garcés $\left(2017\right.$, p.8) $\left({ }^{1}\right)$, describe el contexto del momento actual diciendo que estamos en tiempo de anti-llustración o de analfabetismo ilustrado. Y añade, estamos en "la era de la post-verdad, la época en la que segmentos significativos de la sociedad está dispuesta a creer o a hacer ver que cree en lo que más le conviene en cada momento". Pero esto no sucede espontáneamente.

En efecto, mientras que los individuos hoy tienen acceso inmediato a una ilimitada información, susceptible de ser examinada y combinada, estamos comprobando diversos fenómenos preocupantes. En primer lugar, nos preguntamos, ¿cómo una persona corriente evalúa la información que le llega, cómo la procesa y en qué la transforma? No podemos ignorar que gran parte de las noticias servidas por los medios interesados en ejercer un rol político partidario se comunican en formato de "espectáculo", tal como Guy Debord ya anticipó en $1967\left({ }^{2}\right)$ y Giovanni Sartori en $1997\left({ }^{3}\right)$.

En segundo lugar, millones de personas se han pasado a la mensajería instantánea servida por los canales más populares de internet y a través de sus propias redes sociales y chats. Sin embargo, los canales por los que circulan dichos mensajes son propiedad de oligopolios que, por encima de todo, cuidan de sus 
Re-aprender a pensar. La urgencia de formarse en

el pensamiento crítico como profesionales y como ciudadanos.

Joan Rué Domingo

propios intereses económicos y de dominio del mercado. Si aquellos intereses se ven comprometidos, como en algunos países como China, se restringen determinados datos e informaciones. Si los algoritmos de estas empresas detectan determinadas preferencias en los usuarios, se les ofrecerán ciertas informaciones en detrimento de otras. Por lo tanto, mientras se cree que encontramos lo que buscamos, en realidad se nos ofrece tan sólo aquello que nos gusta.

Hoy ya no podemos sostener la ingenuidad inicial respecto a Internet y a sus operadores, en sus diversas variaciones. Aquellas herramientas que nos publicitaban la apertura al mundo en $360^{\circ}$ desde nuestras propias máquinas, en realidad nos circunscriben dentro de las redes de iguales o pueden exponernos directamente a flujos enormes de falsedades o mentiras. Así se evidenció en Estados Unidos cuando el empleo de las tecnologías informáticas se utilizó para condicionar interesadamente el voto democrático en las elecciones presidenciales. En el Reino Unido también sucedió un fenómeno semejante en relación con el referéndum del Brexit. En Brasil, el nuevo presidente ha sido elegido en votación popular en un contexto electoral en el que los mensajes enviados a través de las denominadas "redes sociales" eran falsos en un $92 \%$. En Hungría, los ciudadanos votaron un jefe de gobierno que sostiene que "el intelecto es improductivo, que (...) habla de "democracia aliberal... El gobierno de mi país demoniza asignaturas y estudios de género, sociales, medio ambiente, inmigración... Los estudios de sociología están siendo limitados" afirma Liviu Matei ${ }^{4}$.

A este respecto, alguien que conoce profundamente el mundo de la industria tecnológica, como Tom Cook presidente de Apple, aporta unos datos concluyentes. En una conferencia en el Parlamento Europeo, en Bruselas el 24 de octubre de $2018\left({ }^{5}\right)$, expresó su alarma sobre "la palabrería política disgregadora que prolifera en las plataformas de las redes sociales" y sobre actores y gobiernos deshonestos que utilizan algoritmos para "profundizar las divisiones, incitar a la violencia e incluso socavar nuestro sentido compartido de lo que es verdad y lo que es falso". Citó al emergente "complejo industrial de datos" que permite a las compañías "conocerte mejor que tú mismo", los cuales se están enriqueciendo con las "reservas de datos 
Re-aprender a pensar. La urgencia de formarse en

el pensamiento crítico como profesionales y como ciudadanos.

Joan Rué Domingo

personales". "Nuestra propia información, desde lo cotidiano hasta lo profundamente personal, se está armando contra nosotros con eficiencia militar", dijo. "Esto es vigilancia, y estas reservas de datos personales solo enriquecen a las compañías que los recopilan". Y "esta crisis es una crisis real, no es imaginaria, ni exagerada ni loca", concluyó.

En este contexto, pensar se convierte en una actividad fundamental, a la vez que urgente. Se trata de pensar como actividad explícitamente consciente, reflexiva y crítica, para ser capaces de entender, de entendernos a nosotros mismos y de entendernos mejor con los demás en el contexto de una democracia abierta. Porque el pensamiento reflexivo es el mejor medio para combatir el propio pensamiento, cuando éste asume fines distintos a la autonomía personal y social. De ahí el sentido de urgencia expresado en el título de este texto.

\section{EL MARCO: MODOS DE VIDA, FORMAS DE PRODUCCIÓN Y CIUDADANÍA DEMOCRÁTICA.}

El diagnóstico que hace Garcés, no obstante, es necesario dotarlo de algunas razones que lo justifiquen, porque siempre pensamos en y desde el marco social e individual en el que nos situamos. Así, encontramos razones vinculadas con la política, con la Inteligencia Artificial y argumentos de orden social y productivo.

Por primera vez en la historia de la humanidad, una misma generación puede ver como el mundo que le vio crecer y desarrollarse alumbra un futuro del que apenas nadie sabe nada. En épocas pasadas, la gente ha experimentado cambios y revoluciones, pero se seguía viviendo de acuerdo con los mismos valores. La información estaba localizada en ciertas personas o instituciones y el acceso a la misma era complicado y demoraba en el tiempo. Los padres y los diversos grupos sociales tenían una idea aproximada acerca de cómo vivirían sus hijos y cómo sustentarían su vida. Los conventos y escuelas parroquiales primero y las escuelas públicas después fueron el lugar privilegiado para acceder a la información disponible, para aprender y reproducir dicho aprendizaje. Las formas de pensar, 
Re-aprender a pensar. La urgencia de formarse en

el pensamiento crítico como profesionales y como ciudadanos.

Joan Rué Domingo

para la gran mayoría de las personas, se circunscribían a lo que se explicaba desde el púlpito, a lo que se aprendía en la escuela y en los textos canónicos y en el trabajo, mediante las costumbre vividas y explicadas en familia. A partir del S. XIX también se aprendía a pensar a partir de la experiencia vivida y lo aprendido en las fábricas y en los ateneos obreros.

Hoy, paradójicamente, la información disponible ha pasado a ser relativamente irrelevante por su universalidad, por su profusión, su ubiquidad y su instantaneidad. Pero también por la falta de filtros. Desde un "simple" teléfono, hoy, se puede acceder a cualquier información desde cualquier lugar. Todo ello ha afectado a las formas de vivir y de producir, así como a nuevas formas de pensar y de hacer. La ingente cantidad de datos ha dado lugar a la Inteligencia Artificial para tratarlos. Puede decirse, sin exagerar, que las tecnologías de la información y comunicación se están convirtiendo en un segundo "medio natural" para el desarrollo humano. El problema de ello es que la falta de filtros ejerce una función formativa involuntaria, pero no menos efectiva, de tal modo que cualquier fragmento encontrado en cualquier lugar deviene útil, todo vale. Por consiguiente. si todo vale, todo es irrelevante. En consecuencia, lo que realmente importa a un sujeto es lo que "yo pienso" y comparto con mis iguales. Ello no deja de ser un fenómeno inquietante y contra el que no es fácil luchar, especialmente desde la cultura formativa dominante.

Dicha transformación ha evidenciado las enormes dificultades con las que se encuentra la educación convencional para dar respuestas adecuadas a la nueva situación. $Y$ efectivamente no cabe duda que asimismo las instituciones educativas están siendo interpeladas como efecto de las nuevas formas de aprender. En otro lugar, (Rué, 2018) $\left({ }^{6}\right)$ he manifestado que la experiencia permite observar cómo en las sociedades plenamente escolarizadas (en las cuales se dedican sumas importantes a la investigación, y donde se argumenta que en la Escuela se enseña a pensar) los rumores más dispares y sin contrastar adquieren categoría de información objetiva, o se divulgan masivamente mensajes con valores como el machismo más recalcitrante, la homofobia, etc. En las redes sociales se observa 
Re-aprender a pensar. La urgencia de formarse en

el pensamiento crítico como profesionales y como ciudadanos.

Joan Rué Domingo

que, ante cualquier hecho medianamente complejo, se suelen registrarse una sarta de tópicos, cuando no de insultos. Todo excepto saber replicar con argumentos y razonarlos. Coincidiendo con la enorme influencia social y política ejercida por el "complejo industrial de los datos" denunciado por Cook, ¿no es relevante también señalar que los ciudadanos tienen un déficit en la formación autónoma del pensamiento? ¿No permite pensar que las personas se acreditan en su formación básica habiendo prescindido de valores básicos para la convivencia y la cooperación, como por ejemplo las consecuencias éticas que comporta la aplicación de todo conocimiento o de la responsabilidad de las propias acciones? ¿Por qué muchas personas se sienten amenazadas por lo que no entienden?

Desde el campo de la formación es urgente reflexionar sobre ello. Quizás el mantra liberal de proporcionar mucha información a los estudiantes para que "pensaran por sí mismos" no fuera más que esto, un mantra y no una realidad objetiva. Bauman (2012, p.41) $\left(^{7}\right)$, formula un diagnóstico pesimista al respecto, "el arte de surfear las superficies ha sustituido el viejo arte de sondear las profundidades. La multitarea es doblemente bienvenida: no sólo acelera el aprendizaje, sino que lo convierte en algo inútil”.

Por primera vez, pues, el centro de gravedad de la formación, tanto la básica como la profesional, se ve desplazado desde la adquisición de información hacia la competencia necesaria para su selección, tratamiento y comprensión. Muchos autores y organizaciones relevantes del mundo educativo están proponiendo un nuevo consenso para la formación, basado en las cuatro "C": pensamiento crítico, comunicación, colaboración y creatividad. A partir de este giro copernicano, la formación debería centrarse menos en las habilidades técnicas para centrase mejor en competencias para adaptarse a "nuevas condiciones y situaciones, a ser capaz de aprender cosas nuevas y de mantener el equilibrio mental ante nuevas situaciones" propone Noha Harari, $\left(2018\right.$, p. 359) $\left({ }^{8}\right)$.

No obstante, a esas aportaciones les falta explicitar una pregunta clave. Cuando no sabemos nada del mañana, sobre cómo se vivirá, en qué se trabajará y 
Re-aprender a pensar. La urgencia de formarse en

el pensamiento crítico como profesionales y como ciudadanos.

Joan Rué Domingo

cómo, tan sólo podemos estar seguros de una cosa. En efecto, la historia nos ilumina sobre dos posibilidades contrapuestas, las personas pueden vivir instrumentalizadas por determinados poderes o bien pueden vivir de modo emancipado y ejercer colectivamente una determinada resistencia o incluso un control sobre los poderes y constricciones que enmarcan su vida. En consecuencia, un argumento que es fundamental, enseñar a pensar a las personas para empoderarlas, para que puedan devenir agentes activos de su propio desarrollo personal y cívico, emerge como un nuevo imperativo ético, formativo y democrático. La innovación exige, de entrada, una cultura del riesgo y de la responsabilidad, afirma Daniel Itinerarity $\left(2011\right.$, p. $229\left(^{9}\right)$ y el aprendizaje institucional debe preparar para ello. La pregunta clave pues es cómo hacerlo.

Este y no otro es el legado del progreso científico y filosófico humanista, a partir del S. XVII. Pensar e investigar son estrategias para liberar a la humanidad de constricciones como las enfermedades, las condiciones de trabajo y de vida, para iluminar a las personas acerca de sus propias debilidades y prejuicios y mostrarles nuevas posibilidades, así como para organizar las sociedades de modo más democrático y entender el mundo desde perspectivas éticas y científicas cada vez más elaboradas y argumentadas. En 1784, en respuesta a la pregunta ¿qué es llustración? Kant $\left({ }^{10}\right)$ recordó que en una sociedad ilustrada las personas se atrevían a pensar por sí mismas.

\section{¿QUÉ ES PENSAR?}

Pensar es imaginar, proyectar, querer, sentir, recordar y hablar. Pensamos porque la realidad nos interpela. Si adoptamos como punto de partida las acepciones del diccionario de la RAE para el verbo pensar nos encontramos con las siguientes cuatro grandes acepciones: a) reflexionar acerca de una acción futura (tener la intención de hacer algo); b) observar, considerar, analizar (examinar mentalmente algo con atención, tener en consideración algo o a alguien al actuar); c) evocar un recuerdo, un conocimiento previo (recordar o traer a la mente algo o a 
Re-aprender a pensar. La urgencia de formarse en

el pensamiento crítico como profesionales y como ciudadanos.

Joan Rué Domingo

alguien); d) elaborar nuevas ideas a partir de hechos, pensamientos, sensaciones, etc (formar o combinar ideas o juicios en la mente, formar en la mente un juicio $u$ opinión sobre algo. Opinar algo acerca de una persona o cosa).

La "reflexividad", es decir, la capacidad de pensar lo que se hace y de pensarse a sí mismo, es la posibilidad de generar proyectos de acción, o proyectos de vida. Constituye uno de los mayores valores humanos. Se afirma que pensar lo hace todo el mundo y que todos podemos alcanzar un pensamiento autónomo. Sin embargo, no todo el mundo piensa de la mejor manera posible, ni lo hace reflexivamente, ni todas las situaciones y contextos lo facilitan o, incluso, lo permiten. Pero tampoco las herramientas y canales de acceso disponibles facilitan el hecho de pensar.

El éxito de las estrategias de la propaganda (además de las enormes sumas destinadas a las mismas y a la geolocalización estratégica de sus destinatarios) es posible porque las ideas, lejos de ser entidades singulares, son nudos en una amplia red o memoria asociativa, en la que cada representación o idea se halla asociada a muchas otras. De modo que, si "alguien" enfatiza, por medio de la propaganda, una determinada conexión, es altamente probable que la idea resultante en los individuos sea la más favorable a la inducción asociativa que se deseó provocar. Es conocido el experimento consistente en que unos sujetos evoquen animales en un tiempo limitado. Cuando la consigna es neutra los sujetos enumeran muchos más animales que cuando el instructor les pide que nombren animales, pero, por ejemplo, que eviten nombrar al elefante.

Ciertamente todos los humanos estamos capacitados para pensar. No obstante, existen tres fenómenos importantes de singular relevancia para cualquier formador que desee estimular y favorecer en sus alumnos un pensamiento más autónomo:

En primer lugar, la psicología moderna ha destacado la supeditación del hecho de pensar a una razón de orden a la vez emocional y económico, vinculada al interés y a la economía del esfuerzo individual. ¿Me interesa? ¿Compensa pensar? 
Re-aprender a pensar. La urgencia de formarse en

el pensamiento crítico como profesionales y como ciudadanos.

Joan Rué Domingo

De acuerdo con Kahneman, (2012, p. 54) $\left({ }^{11}\right)$ la ley del mínimo esfuerzo establece que "si hay varias formas de lograr el mismo objetivo el individuo gravitará finalmente hacia la pauta de acción menos exigente. En la economía de la acción el esfuerzo es un coste y la adquisición de habilidad viene determinada por el balance de costes beneficios. La pereza está profundamente arraigada en nuestra naturaleza" afirma.

El escritor israelí Noha Harari (2018, p.25) lo formula de un modo distinto, pero coincidente con la tesis anterior: "Los seres humanos piensan en historias por encima de hechos, números o ecuaciones y cuanto más sencilla sea la historia, mejor". Y si estas historias se nos dan hechas (sin profundizar en sus aspectos discutibles o improbables, $u$ ocultándolos) y aparentando reunir todas las posibles explicaciones, las personas lo adoptarán hasta donde sea necesario porque "ofrecen un puerto seguro contra la frustrante complejidad de la realidad (p.319).

En segundo lugar, toda forma de pensar se halla imbricada siempre en una matriz sociocultural construida y aprendida por los individuos. En efecto, todo aprendizaje individual es la síntesis de los múltiples procesos interactivos de naturaleza cooperativa en los que se involucra una persona, con todos los diversos agentes de su entorno. $Y$ es conocida la dificultad de sustraerse a las opiniones dominantes en el propio entorno social, tanto por el argumento de la "facilidad" anterior como por nuestra tendencia al gregarismo, es decir, a mantenernos aceptados entre los iguales.

Finalmente, se da la posibilidad de que las personas podemos pensar tanto de un modo superficial como de modo más profundo. Un pensamiento más superficial sería aquél conectado con el plano de la naturaleza técnica de la acción, el que trata de responder al cómo, al cuándo o al con qué. Por el contrario, una mayor exigencia reflexiva es la que nos propone pensar acerca de las propiedades de un fenómeno: el qué, sus características, su diagnóstico. $Y$ de un modo especial, sobre las finalidades de algo o de una acción, así como sus exigencias éticas (los para qué, los por qué), es decir a todo aquello que remite a las consecuencias y responsabilidades de una determinada acción. A mayor profundidad es necesario un 
Re-aprender a pensar. La urgencia de formarse en

el pensamiento crítico como profesionales y como ciudadanos.

Joan Rué Domingo

mayor esfuerzo, pero en correspondencia el individuo alcanzará una mayor autonomía.

En su extremo una secta, el pensamiento sectario, cumple plenamente con la primera y segunda de estas características, lo que permite que los sujetos se planteen tan sólo cuestiones de orden procedimental o técnico en su conducta sectaria. En otro ejemplo menos extremo, las conductas de masas también pueden explicarse mediante estos mismos mecanismos. Sin embargo, aunque distantes, ambas formas de conducta comparten un rasgo en común, la desresponsabilización y ausencia de análisis reflexivo de los agentes sobre sus propias conductas.

\section{EL RAZONAMIENTO, SUS MÚLTIPLES VÍAS Y SUS LIMITACIONES}

La psicología actual asume dos grandes principios que si bien parecen obviedades no lo son, porque en la práctica formativa no se explotan adecuadamente. Por una parte, sabemos que los seres humanos somos creadores de realidades; sabemos que tenemos la posibilidad de desarrollar sensaciones, percepciones, sentimientos, ideas, intuiciones, sueños, anhelos, deseos, y que todos estos factores son una parte importante de nuestras reflexiones (Sastre, 2018) $\left({ }^{12}\right)$. Por otra, hemos visto que las realidades físicas, materiales y culturales en las que nos desenvolvemos los individuos, configuran el marco desde el que pensamos y desde el que creemos que el hecho de pensar es útil o necesario. El primero de estos principios nos ilumina acerca de las enormes posibilidades del pensar. El segundo, por el contrario, lo circunscribe, para cada individuo, a un escenario relativamente limitado de posibilidades.

Sin entrar en explorar situaciones limitadoras del pensamiento, como es el caso de la pobreza o de la escasez de recursos, de tiempo, etc. (Shendil, Shafir, 2016) $\left({ }^{13}\right)$, en el caso particular de la formación impartida en los centros formativos, las condiciones ordinarias bajo las que se imparte la enseñanza, la presión para el desarrollo de los programas o las formas de evaluación ejercen una fuerte presión sobre unos tipos de razonamientos en detrimento de otros. 
Re-aprender a pensar. La urgencia de formarse en

el pensamiento crítico como profesionales y como ciudadanos.

Joan Rué Domingo

Así, en las situaciones de formación suele primarse al razonamiento convergente sobre el divergente. El desarrollo del razonamiento lógico suele preferirse al creativo o al divergente, olvidando que la lógica por sí misma es un recurso formal, si no hay un agente que la active con un fin o intencionalidad determinada. ¿Es el razonamiento lógico o el creativo el que da ventaja a un jugador sobre otro en el ajedrez, por ejemplo? Lo que realmente da ventaja, ¿no es una mejor intuición, una forma divergente de razonar?. No obstante, desde los modelos de formación convencionales se suele enseñar más a responder de acuerdo con una expectativa esperada que a aplicar un razonamiento interrogador o a elaborar las mejores preguntas ante una situación que no se domina. También se favorece al pensamiento deductivo por encima del inductivo.

Sin embargo, la búsqueda de analogías, el razonamiento creativo y un pensamiento sistémico se manifiestan como indispensables cuando la formación se ofrece en términos de problema: es decir, en el aprendizaje basado en problemas $(A B P)$, por ejemplo; porque los problemas se le ofrecen al aprendiz en clave de complejidad en la que convergen elementos del contexto, aspectos técnicos, incidencia de factores cuyo origen pueden tener campos disciplinarios diversos. Todo ello, a su vez, interpela al sujeto que aprende en toda la complejidad de su experiencia y un mayor repertorio de recursos mentales. Ciertas formas de enseñanza y aprendizaje, pues, ejercen una fuerte restricción sobre el ejercicio del pensamiento privilegiando unas áreas mentales determinadas en lugar de promover un ejercicio sistémico del pensamiento.

Esta concepción restrictiva del pensamiento en la enseñanza supera a los propios profesionales, porque se halla condicionada por una cultura escolar clasificadora y acreditadora, de carácter fundamentalmente técnico. Ello se observa en la fragmentación de materias y tiempos, en el diseño individualizador del mobiliario y de los espacios, o en el modo cómo se enfocan las evaluaciones y se obtienen las valoraciones o notas. Éste es uno de los mayores logros de la incorporación del taylorismo a la enseñanza, y con él las representaciones ideológicas que lleva asociadas. Unas representaciones que no tienen 
Re-aprender a pensar. La urgencia de formarse en

el pensamiento crítico como profesionales y como ciudadanos.

Joan Rué Domingo

suficientemente en cuenta que "los alumnos" son "personas" que van configurando su forma personal de actuar, de pensar y de sentir en interacción con su amplio entorno cultural. El poder de convicción de este modelo ha sido tan enorme que ha persistido, a pesar de los resultados contrarios de la investigación psicopedagógica y del hecho de contradecir las experiencias comunes de las personas (Rué, 2018).

Se olvida que para modificar la conducta es necesario formar a las personas para que cambien sus modelos generales de razonar ante diversos retos cognitivos. Precisamente las aportaciones de la psicología moderna muestran las limitaciones de nuestras mentes en la forma de operar. A veces, se "piensa" sólo desde las emociones. O desde una visión inmediata del aquí y ahora, o sólo desde el marco creado por otros. Ello revela la necesidad de utilizar metodologías docentes que favorezcan la generalización de conocimientos a contextos cada vez más amplios y más alejados de las situaciones concretas en las que el aprendizaje inicial tuvo lugar.

En efecto, se piensa mejor, con más sentido y de modo reflexivo, cuando se tienen esquemas y herramientas para ello, cuando las personas se han entrenado suficientemente en ello, cuando las emociones no ocupan todo el escenario cognitivo, cuando se usan estrategias y esquemas apropiados - que deben ser aprendidos - y se emplean conscientemente, es decir, cuando se sabe por qué y para qué se hace lo que se hace y con qué se hace.

Se puede decir, con cierta razón, que la escuela ya realiza este tipo de formación mediante la enseñanza de las ciencias y las tecnologías. A pesar de ser cierto, este argumento es parcial, porque en la formación suele darse por supuesto que las personas razonan suficientemente y lo hacen sin demasiados sesgos. Pero ello no es así. Las personas cometemos errores, malinterpretamos informaciones, no aceptamos evidencias y emitimos diagnósticos erróneos. Ello no es así porque no seamos suficientemente listos o aplicados. Simplemente porque está en nuestra naturaleza cometer este tipo de errores. Una "naturaleza", por lo tanto, que debe ser reflexionada a su vez y ser también educada. 
Re-aprender a pensar. La urgencia de formarse en

el pensamiento crítico como profesionales y como ciudadanos.

Joan Rué Domingo

Veamos algunos ejemplos de diversos tipos de efectos que nos condicionan significativamente sin que tengamos una conciencia clara de ello. Exceptuado los dos últimos, los ejemplos están extraídos de Kahneman (2012):

- Los efectos del cansancio, en concreto de la falta de glucosa, en la toma de decisiones, por ejemplo. En esta situación, los errores intuitivos son normalmente mucho más frecuentes. En una tarea que se les daba a dos grupos de personas se dio una diferencia significativa entre ellos en su ejecución. El grupo que se mantuvo agotado (sin darles glucosa) obtuvo un índice elevado de deterioro de la tarea. En otro trabajo, los autores analizaron las decisiones que tomaron jueces de los tribunales, cansados y después de horas sin comer. Observaron que, en estas condiciones, tendían a tomar la decisión más fácil y común de denegar peticiones de libertad condicional (p. 63-64). Esta es una observación susceptible de ser generalizada a muchas otras profesiones en condiciones semejantes y a la toma de decisiones en muchas reuniones.

- Confiar en exceso en la intuición. Kahenman (p. 65-66) lo ilustra mediante un problema sencillo. A los estudiantes se le propone la cuestión siguiente:

Un bate y una pelota juntos cuestan 1,10 dólares

El bate cuesta un dólar más que la pelota

¿Cuánto cuesta la pelota?

Mientras que la respuesta son 5 centavos, más del 50 por ciento de estudiantes de Harvard, del MIT y de Princeton dieron una respuesta intuitiva incorrecta. En universidades menos selectivas el número de respuestas incorrectas alcanzaba el $80 \%$. La intuición nos confunde: jes muy fácil!

- La atención selectiva. Podemos estar ciegos para lo evidente y ciegos, además, para nuestra ceguera, afirma el autor mencionado. En un experimento de Simons y Chabris 1999, que puede seguirse en youtube $\left({ }^{14}\right)$, se pregunta a los observadores que cuenten el número de pases que se hacen los jugadores con una 
Re-aprender a pensar. La urgencia de formarse en

el pensamiento crítico como profesionales y como ciudadanos.

Joan Rué Domingo

pelota. De repente, un gorila se pasea por la cancha durante unos segundos. Muchos observadores ignoran por completo la presencia del gorila.

- El control ejercido sobre la atención (p.68). Mischel y sus estudiantes dieron a elegir a niños de cuatro años entre una recompensa simple que podían obtener en cualquier momento y una recompensa mayor (dos galletas), para la que tenían que esperar 15 minutos, en condiciones difíciles y en solitario en una habitación. Alrededor de la mitad de niños esperaron 15 minutos desviando la atención de la tentación de la recompensa. Años más tarde se había abierto una enorme brecha entre los que habían resistido y los que no. Los que esperaron, a los cuatro años más tarde dieron mejores resultados en ejecución de tareas cognitivas y en rendimiento en los test de inteligencia. Otra investigación del mismo grupo relacionó el déficit y el control de la atención con métodos de crianza.

- La intensificación en el trabajo. Pasar de una tarea a otra requiere esfuerzo, especialmente cuando el tiempo apremia (p. 56).

- La percepción puede estar sesgada por el efecto "halo", debido a las emociones suscitadas por el estímulo. (p. 113). En un experimento se presentaban a los sujetos descripciones de dos personas, las cuales eran definidas del modo siguiente:

Alan: inteligente-diligente-impulsivo-crítico-testarudo-envidioso

Ben: envidioso-testarudo-crítico-impulsivo-diligente-inteligente

Cuando a los participantes en el experimento se les preguntó si era plausible que los seis adjetivos describieran a la misma persona, la mayoría pensó que era imposible.

- El efecto marco. Maneras diferentes de presentar la misma información provocan reacciones de percepción distintas. El ejemplo anterior lo ilustra. Otro ejemplo del mismo autor, aplicado al campo de la medicina, también es ilustrativo (p.120): un paciente estará más tranquilo si se le dice que "las posibilidades de supervivencia después de un mes de la operación son del el $90 \%$ "que si se le dice que la mortalidad después de un mes de la operación suele ser de un $10 \%$ ". 
Re-aprender a pensar. La urgencia de formarse en

el pensamiento crítico como profesionales y como ciudadanos.

Joan Rué Domingo

- El propio sistema de creencias. Los hechos que desafían asunciones básicas, medios de vida, autoestima de las personas, simplemente no son asimilados. La mente no los digiere (p. 283). La gente tiende a recabar datos que avalen o sean compatibles con sus creencias previas. "Sabemos que las personas pueden mantener una fe inquebrantable en una afirmación, por absurda que sea, cuando se sienten respaldadas por una comunidad de creyentes de su misma mentalidad" (p. 285). La confianza de las personas en una creencia se basa en dos impresiones: la facilidad cognitiva y la confianza (p. 313) El pensamiento asociativo está hecho para suprimir la duda y suscitar ideas e información que sean compatibles con la historia dominante en el momento.

- La propensión de nuestro cerebro por el orden. Motterlioni (2010, p57-58) $\left({ }^{15}\right)$ comenta que nuestra obsesión por el orden "nos ayuda a manejar con más facilidad el enorme flujo de informaciones que nos envuelve" (...) Pero esta propensión nos hace caer en la superstición y en las lecturas míticas de la realidad" El orden que a veces imaginamos, "sus estructuras son tan sólo producto de nuestra mente" (...) Nuestra convivencia con el azar es difícil ... El asombro por la frecuencia de hechos raros nos induce a abandonar la lógica de las leyes de la probabilidad para encontrar explicaciones de distinta naturaleza".

- La percepción de la facilidad de una idea, apoyada en el gregarismo. La reducción de un hecho a un titular, a unos pocos caracteres, elimina el contexto, reduce el hecho simplificándolo y ahorra pensar al receptor. El apoyo de los "likes", además, elimina cualquier sentido de responsabilidad con respecto a la necesidad de matizar y a la simplificación enunciada. Ello suele eliminar los grises, los matices de cualquier idea y los tópicos y estereotipos se adueñan del pensamiento, según se observa en el nuevo binomio internet-lenguaje político. El resultado es un pensamiento binario empobrecedor $y$, a menudo, embrutecedor. 
Re-aprender a pensar. La urgencia de formarse en

el pensamiento crítico como profesionales y como ciudadanos.

Joan Rué Domingo

\section{EL PENSAMIENTO CRÍTICO ES EDUCACIÓN}

El pensamiento es la principal herramienta de liberación. El lema kantiano de la llustración, el Sapere aude, "atrévete a pensar por ti mismo", era, y sigue siendo un deber y un reto. El hecho de pensar crítica y seriamente tiene pretensión de universalidad, para cualquier persona, en cualquier lugar y tiempo, afirma Begoña Román (2018) $\left({ }^{16}\right)$.

A diferencia del pensamiento común, el pensamiento crítico es más exigente. El adjetivo crítico deriva del griego, cuya etimología significa cribar, filtrar y, a su vez, está emparentado con "crisis". Esta referencia, por lo tanto, nos remite a una crisis en el pensamiento, podríamos decir, causada por la perplejidad, por la paradoja, la cual obliga a distinguir, a dirimir para elaborar un mejor diagnóstico o apreciación. Y la actividad de distinguir, clasificar y dirimir, exige también de un cierto rigor. Aquel tipo de crisis en el pensamiento Piaget lo denominó conflicto cognitivo, y lo consideraba como un suceso necesario para progresar en la elaboración de una idea o en su profundización. Así, desarrollar el pensamiento crítico supone aprender a realizar relaciones pertinentes. El matemático Henry Poincaré decía que crear es escoger las combinaciones útiles, muy escasas, de entre las inútiles, abundantes en demasía, (en Albertí, 2011, p.29) $\left({ }^{17}\right.$ )

La noción de pensamiento crítico emerge, en la actualidad, con un nuevo énfasis en la cultura educativa. Surge como contraposición a una forma habitual de pensar en educación, sometida a la regla de la lógica del conocimiento elaborado y ofrecido para su asimilación acrítica. El desarrollo de la cultura educativa ha sufrido así un serio desequilibrio. Los currículos oficiales elaborados, los tiempos disponibles para su asimilación, las condiciones para ello y las modalidades más habituales de evaluación y de acreditación, han priorizado históricamente un pensamiento escolar adaptativo en el que pensar significaba fundamentalmente seguir la lógica de lo expuesto y razonar a partir del marco de contenidos librado desde la palabra de los docentes o por el texto canónico empleado. Las formas de control escolar han devaluado la asimilación de otros tipos de pensamiento, el crítico entre ellos. En contraposición, el pensamiento moral, el para qué, el que reflexiona 
Re-aprender a pensar. La urgencia de formarse en

el pensamiento crítico como profesionales y como ciudadanos.

Joan Rué Domingo

sobre las consecuencias de una determinada lógica, el que se interroga por los marcos desde los que se piensa, el que trata de contextuar para realizar una mejor valoración, han quedado relegados a un segundo plano en la escolaridad.

Una razón para entenderlo es que el principio del rendimiento, aplicado sobre los actuales currículos y programas saturados, convierte al proceso formativo en una carrera de obstáculos para los formados. En este proceso se suelen pedir entregas de trabajos, superponiéndose a menudo entre sí, en los cuales los estudiantes deben buscar la respuesta adecuada sin dedicar el tiempo necesario para pensar estrategias, o para reflexionar sobre lo que se hace, o simplemente para preguntarse por otras opciones y, sobre todo, tiempo para aprender a aprender desde sus propias formas de confrontar el conocimiento. En la noción de rendimiento escolar o académico, tal como suele ser empleada, late una dimensión relativamente limitada del conocimiento, su dimensión utilitarista. De ahí que, cuando los estudiantes no ven la utilidad del mismo, ejerzan aprendizajes meramente formales o directamente acudan a las diversas formas de plagio (Rué 2018), una actitud primaria que podemos entender como defensiva o de supervivencia, pero nada ética.

La nueva revaloración del concepto de crítica expresa la encrucijada en la cual se encuentra la formación en la actualidad. Por una parte, profundizar en la vía tecnoliberal, es decir, aquella que tiende a la desconexión entre la técnica y la conciencia, la que separa el qué y el cómo de toda reflexión ética y de todo propósito. O, por otro lado, revalorizar el desarrollo de un conocimiento tecnohumanista inspirado por el viejo aforismo inscrito en el pronaos del templo de Apolo en Delfos en el S. IV a. C., "conócete a ti mismo". En este escenario, la tríada autonomía, pensamiento crítico y compromiso ético son primordiales en educación, sostiene Román (2018). En consecuencia, podemos decir que el desarrollo del pensamiento crítico supone aspirar a alcanzar la fase más elaborada de lo que entendemos por educación. 
Re-aprender a pensar. La urgencia de formarse en

el pensamiento crítico como profesionales y como ciudadanos.

Joan Rué Domingo

\section{IR MÁS ALLÁ DE LAS LIMITACIONES DE LA VIGENTE CULTURA ESCOLAR}

Un espacio privilegiado para aprender a pensar críticamente es el de la formación. En sus diversos escenarios y niveles. Tanto por ta razón de contenidos (los cuales deben comprenderse para asimilarlos), como porque las situaciones de enseñanza y aprendizaje se producen en contextos sociales dialógicos, donde se puede interaccionar y reflexionar conjuntamente con los demás a partir de las propuestas de aprendizaje.

Sin embargo, la particularidad de nuestro sistema formativo, generado en el contexto sociocultural de la era industrial, es que en el se han ido generando unas representaciones, unos contextos de trabajo y unas prácticas incrementadas progresivamente de contenidos y técnicas, cuya asimilación es exigida por el mismo sistema. La singularidad de dicha asimilación es que en ella se dirime el ser reconocido por el mismo sistema, es decir, para acreditarse las personas en ránquines que los individualizan. Los déficits de este sistema se ponen de manifiesto en la sociedad del conocimiento, o en los inicios de la era del Antropoceno, cuando esta cultura formativa se pone en crisis (Rué 2018), por lo que debe ser revisada para ir ajustándose a los requerimientos de la vida en democracia y de las actividades humanas emergentes.

Sastre (2018), con acierto, afirma que una de las cuestiones importantes del momento actual de la educación es formar "a las nuevas generaciones con marcos de pensamiento que reflejen la unidad y diversidad de las facetas del mundo en el que vivimos". Ahora bien, aunque el sistema formativo no puede - por sí mismo reconvertir la situación histórica descrita, sin embargo, debe alinearse con las fuerzas que quieren ser parte de la solución y no formar parte del problema. De ahí que hay que dirimir sobre qué saberes y qué prácticas culturales necesitamos elaborar, desarrollar y compartir en cualquier formación para que contribuyan a otro modelo educativo, el de empoderar a los ciudadanos en el ejercicio de su libertad de pensar. 
Re-aprender a pensar. La urgencia de formarse en

el pensamiento crítico como profesionales y como ciudadanos.

Joan Rué Domingo

En este sentido, es fundamental empezar reconociendo la dificultad que implica pensar críticamente. Por ello, como tantas otras actividades humanas, requiere un aprendizaje determinado y constante a lo largo de toda la vida. En segundo lugar, para que dicha formación tenga un efecto determinado, es necesario revisar algunas de las prácticas formativas que se han acabado por naturalizar. Así, por ejemplo, no es lo mismo una formación que prioriza el pensar a aquella que propone seguir el ritmo del profesorado. Tampoco lo será un proceso en el cual las personas sean consideradas instrumentales de un programa y de unos resultados preestablecidos que otra formación orientada desde el principio a que el estudiante sea el propio fin de la misma. A partir de estas dos asunciones básicas, en la práctica de enseñar a pensar críticamente propongo considerar las siguientes cuestiones:

1. La mayor libertad de las personas, su independencia para pensar, tiene como contrapartida que aprender y autoeducarse para vivir más dignamente es costoso. Requiere constancia, esfuerzo y apoyo. El ejercicio continuado de la voluntad y el esfuerzo de pensar por uno mismo supone hacerlo siguiendo unas pautas de racionalidad o una cierta metodología para que quien la ejerza vea su provecho. Finalmente, requiere también socializarse en el pensar, mediante el diálogo. Se piensa con los demás y se piensa mejor en una relación dialógica cooperativa, tal como ha mostrado el socioconstructivismo y, más en concreto, el corpus de investigación sobre el aprendizaje en cooperación $\left({ }^{18}\right)$.

2. Pensar críticamente es costoso porque, como toda buena formación, desafía el sentido de normalidad, entendida como el pensamiento común y socializado sobre algo. Pero también por lo que supone de desafío al pensamiento emocional e intuitivo. Más arriba hemos visto ejemplos de condicionantes del pensamiento, profundamente enraizados en la naturaleza humana. En ocasiones el pensamiento desafía modalidades más elaboradas del mismo, lo que expresa nuestra querencia hacia las narraciones simples y cerradas, a los 
Re-aprender a pensar. La urgencia de formarse en

el pensamiento crítico como profesionales y como ciudadanos.

Joan Rué Domingo

dogmas o a nuestra tendencia a la economía, a los prejuicios y a la tentación del pensamiento prêt a porter.

3. El pensamiento crítico desafía al concepto de "idea", tal como es presentado en los currículums formativos. Lejos de verlas como algo acabado, listas para ser consumidas y asimiladas, las ideas tienen su historia, son como un trabajo de bricolaje, construidas a partir de restos e imputs dispares. $Y$ así deberían ser expresadas, porque es necesario aprender que el pensamiento siempre está necesitado de préstamos, de experiencias, de referentes y de puntos de vista de otros. En oposición a una visión escolar simple, hemos visto más arriba que las ideas se conciben como nudos de redes más amplias, de ahí que aprender sea un continuo del que siempre parece que algo se escapa. Así, formarse en el pensamiento crítico, requiere formarse en la complejidad y la diversidad, de puntos de vista, de enfoques y de percepciones.

Johnson (2011, p..57) $\left({ }^{19}\right)$ comenta que "para que una mente sea más innovadora hay que colocarla en entornos que en sí mismos sean también redes: entornos de ideas o de personas que imiten las redes cerebrales de la mente que explora los límites de lo posible adyacente. Ciertos ambientes fomentan la capacidad natural del cerebro para crear vínculos nuevos. Una idea es una red. Pero necesita: que esté densamente poblada y que sea plástica, capaz de adoptar configuraciones nuevas, comunicarse de forma distinta y adoptando conexiones también distintas. $Y$ añade: "La forma más rápida de congelar una red es meter a la gente en despachos privados, con la puerta cerrada", (p.39).

4. El pensamiento crítico desafía las categorías simples y los dualismos, siempre empobrecedores. Se ha querido contraponer razón y emociones, como una derivación de los mundos material y espiritual, como si fueran dos opciones distintas cuando una y otra se entremezclan en cada actividad. La razón controla a la emoción y la emoción incita a la razón. ¿Cómo distinguirlas?

5. El pensamiento tiene como objeto la finalidad de entender y actuar. De ahí que deba vincularse el conocimiento a una necesidad. El conocimiento que un 
Re-aprender a pensar. La urgencia de formarse en

el pensamiento crítico como profesionales y como ciudadanos.

Joan Rué Domingo

estudiante tiene a su disposición no es un corpus cosificado y cerrado (tal como se le presenta en los diversos currículos enseñados), sino el resultado de muchas inquietudes, de preguntas pertinentes ante necesidades que ciertas culturas y personas debieron afrontar en un momento histórico en un lugar determinado. $Y$ este aspecto del conocimiento suele quedar velado. De ahí, si se desea potenciar el pensamiento crítico, es fundamental incitar a los formados a observar, a considerar lo sorprendente e interpretar, haciendo hipótesis y conjeturas, así como enseñar a hacer buenas preguntas, a buscar analogías, a seguir intuiciones y explorarlas, a buscar pruebas y a refutarlas, si es necesario.

6. Asumir el error ... y provocarlo. Contrariamente a las valoraciones de la escuela acreditadora sobre el mismo, el error constituye un elemento fundamental en el progreso del conocimiento. Steven Johnson (p.149) cita al matemático y economista inglés William Stanley Jevons, quien en sus Principles of Science, en 1874, decía: "Con toda probabilidad, los errores de las grandes mentes exceden en número al de las mentes menos vigorosas. Entre los primeros requisitos para descubrir algo están la fertilidad de la imaginación y la abundancia de conjeturas; pero las conjeturas erróneas deben ser mucho más numerosas que las que demuestren estar bien fundadas" (p.174). En un sentido complementario, Johnson también cita a Charlan Nemeth, (p. 153-154), quien en sus experimentos sobre la creatividad observó que las respuestas humanas tienden a ser esperables y sólo alrededor del $20 \%$ de las personas da respuestas de naturaleza divergente. Ahora bien, cuando a los sujetos dentro del $80 \%$ de respuesta tópica se les induce al conflicto cognitivo mediante una respuesta errónea, el conjunto de ellos daba respuestas más creativas.

7. La creación y sostén de un entorno actitudinal adecuado es fundamental. Actitudes éticas como las de compromiso y persistencia en la tarea, la del rigor en el habla, en la observación, en la toma de notas, en la clasificación, en la organización de una secuencia lógica, en la selección y seguimiento de un 
Re-aprender a pensar. La urgencia de formarse en

el pensamiento crítico como profesionales y como ciudadanos.

Joan Rué Domingo

orden dado en la acción, deben desarrollarse en el mismo proceso de aprender a pensar. No puede darse el segundo sin las primeras.

8. Saber contextuar la información y situarla en términos históricos. Ningún dato es ajeno a determinadas causas, ni se explica suficientemente sin referentes. Todo valor remite a unas expectativas determinadas, a fenómenos como la escasez o la abundancia, a la oferta o a la demanda. La experiencia pasada, si es relevante, pueden ser los hombros sobre los que actualmente viajamos.

9. Promover el pensamiento social. Las humanidades y las ciencias sociales en la formación configuran un espacio formativo que nos remite a las formas de expresión más genuinamente humanas. Tales como la libertad, la interacción cooperativa con los demás, la empatía, la reflexión. $O$ el hecho de aprender a afrontar con espíritu democrático aquellos puntos de vista que nos retan o, incluso, que nos provocan. Aquellos conocimientos son los únicos que tienen el potencial para enriquecer el hecho de hablar y hacer entender el valor del compromiso en una vida en común. Hay que insistir en que la democracia, su calidad en último término, se sustenta en la educación.

10. Leer, aprendiendo de la experiencia de otros. La escritora iraní Azar Nafisi (2014) $\left({ }^{20}\right)$ nos recuerda que una actividad aparentemente tan ordinaria como leer promueve la imaginación, despertándonos la curiosidad hacia realidades que difícilmente podríamos conocer de otro modo. Permite observar a los demás en su complejidad, identificarnos con ellos y generar empatía hacia ellos. La lectura nos permite resolver un obstáculo formidable, entender hechos y situaciones que de otra manera quedarían fuera de nuestro alcance y comprensión. Mediante la lectura se promueve la empatía, la capacidad de escuchar al otro y de aprender a comunicar todas las ideas.

\section{Joan Rué}

Pedagogo. Profesor Universitat Autònoma de Barcelona.

Director de la Revista Catalana de Pedagogia.

Societat Catalana de Pedagogia, IEC

Joan.rue@uab.cat 
Re-aprender a pensar. La urgencia de formarse en

el pensamiento crítico como profesionales y como ciudadanos.

Joan Rué Domingo

${ }^{1}$ Garcés, M., (2017). Nova il/lustració radical. Barcelona, Anagrama.

2 Debord, G. (1976). La sociedad del espectáculo, Madrid, Miguel Castellote.

${ }^{3}$ Sartori, G. (1997). Homo videns. La sociedad teledirigida, Barcelona, Taurus.

4 Liviu Matei, vicepresidente de la Universidad Centroeuropea de Budapest, La Contra, La Vanguardia, 26 de octubre de 2018.

${ }^{5}$ Cook T., (2028). Disponible en: https://www.youtube.com/watch?v=kVhOLkls20A\&feature=youtu.be

${ }^{6}$ Rué, J. (2018). Propuestas para un nuevo pacto sobre educación, Barcelona, Octaedro.

${ }^{7}$ Bauman, Z., (2012). Esto no es un diario, Barcelona, Paidós.

${ }^{8}$ Nora Harari, Y., (2018). 21 lecciones para el S. XXI. Barcelona, Eds. 62.

9 Itinerarity, D., (2011). La democracia del conocimiento, por una sociedad inteligente, Barcelona, Paidós.

${ }^{10}$ Kant, I., (1985). ¿Qué es Ilustración?, México D.F. Fondo de Cultura Económica.

${ }^{11}$ Kahneman, D., (2012). Pensar rápido, prensar despacio, Barcelona, Debate.

12 Sastre, G. (2018). Encrucijadas sociales y educación del pensamiento. Revista Catalana de Pedagogia, 14, 47-66.

${ }^{13}$ Sendhil, M., Shafir, E., (2016). Escasez ¿por qué tener poco significa tanto?, México, FCE.

${ }^{14} \mathrm{En}$ https://www.youtube.com/watch?v=vJG698U2Mvo

${ }^{15}$ Motterlini, M., Trampas mentales, (2010). Barcelona, Paidós.

16 Román, B. (2018). Aprendre a pensar: una mirada des de la Filosofia. Revista Catalana de Pedagogia, 14, 15-26.

${ }^{17}$ Albertí, M., (2011). La creatividad en matemáticas, Barcelona, RBA Editores.

${ }^{18}$ Véase por ejemplo:

- Tomasello, M., (2014). A Natural History of Human Thinking, Cambridge, Ma, Harward University Press.

- Alexander, R., (2005). Education, Culture and Cognition: intervening for growth, UK, CULTURE, DIALOGUE AND LEARNING: NOTES ON AN EMERGING PEDAGOGY Keynote. International Association for Cognitive Education and Psychology (IACEP) 10th International Conference, University of Durham, UK, 10-14 July 2005. http://lpuae.pbworks.com/w/file/fetch/47478116/Dialogic\%20teaching.pdf (Consultado el 29/05/2018)

- Hutchins, E., (2000) Distributed Cognition, IESBS Distributed Cognition. En, http://comphacker.org/pdfs/631/DistributedCognition.pdf 18/05/2018

- Johnson, D, y Johnson, R., (1992). Encouraging thinking through constructive controversy, en Davidson, N., Worsham, T., (eds). Enhancing Thinking Through Cooperative Learning. New York, Teachers College Press.

- Solomon, R.D., Davidson N., y Solomon E.C.L., (1992). Some thinking skills and social skills that facilitate cooperative learning. En Davidson, N., Worsham, T., (eds). (1992). Enhancing Thinking Through Cooperative Learning. New York, Teachers College Press.

19 Johnson, S., (2011). Las buenas ideas. Una historia natural de la innovación. Madrid, Turner.

${ }^{20}$ Nafisi, A., (2014). Leer Lolita en Teherán, Barcelona, Duomo Ediciones.

\section{Cómo citar este artículo:}

Rué, J., "Re-aprender a pensar. La urgencia de formarse en el pensamiento crítico como profesionales y como ciudadanos", Folia Humanística, 2019 (11): 6-28. Doi: http://dox.doi.org/10.30860/0047.

(C) 2019 Todos los derechos reservados a la Revista Folia Humanística de la Fundación Letamendi Forns. This is an open access article. 